\title{
Supernova almost on the doorstep
}

\section{Last week's discovery of a supernova outburst in the Larger Magellanic Cloud is an important opportunity for astronomers of several kinds, but one chance has been missed already.}

Astronomers have been handed a potential treasure trove with the discovery last week of a supernova 155,000 light years away in the nearby satellite galaxy, the Larger Magellanic Cloud (LMC). The exploding star, 10,000 times brighter than any other object in the LMC, provides not only an unprecedented opportunity to study the development of a supernova, but also a beacon by which interesting constituents of the intervening matter can be studied. Perhaps most significantly of all, neutrinos emitted by the initial explosion are thought to have been detected. Not surprisingly, every observatory within sight of the object (predominantly in the Southern Hemisphere) and every appropriate satellite has been rescheduled to monitor the event.

Three astronomers share the credit for the discovery on 24 February. Ian Shelton, of the University of Toronto, found the very bright object by good luck on a three-hour exposure taken at the Las Campanas Observatory in Chile. Oscar Duhalde, a night assistant at the same observatory, spotted the source with the naked eye, as did Albert Jones, an amateur observer of variable stars living in New Zealand.

It turns out that this patch of sky had been photographed by other observers over preceding nights, from which it seems that the supernova was first readily observable (at magnitude 6) on 23 February. Since then, it has brightened by about two magnitudes and is expected to reach its peak brightness within a few days. Meanwhile, the object has been detected at ultraviolet, radio and, very tentatively, $\mathrm{X}$-ray wavelengths and has been granted the inspiring title 1987a.

The neutrino detection was announced by $\mathrm{C}$. Castagnoli and other members of an Italian/Soviet collaboration working at the Mont Blanc neutrino observatory. Five neutrino pulses of energies above 7 $\mathrm{MeV}$ were observed at 0300 Universal Time on 23 February - nine hours before the first optical detection.

Happily, and for the first time, it has also been possible to pinpoint the star whose demise has so brightened the lives of astronomers. The object appears to be the blue B-type supergiant Senduleak -69 202 with a mass between 30 and 50 times that of the Sun. This identification agrees well with the notion that, at the end of their nuclear-burning lifetimes, stars of more than a few solar masses undergo a catastrophic transition, shedding much if not all of themselves in the form of a rapidly expanding shell of gas and often, if not always, leaving behind a compact remnant such as a neutron star. Although there are many such stars in our Galaxy, their explosive ends, reckoned to occur on the average once every 50 years, are obscured - like many of the interesting features of the Galaxy - by gas and dust. Thus astronomers have generally had to make do with such explosions detected in other galaxies.

The new supernova is sufficiently close to offer the prospect of resolving the expanding shell within only weeks of the outburst, although the maximum angle of ten milliseconds of arc that will be subtended by the bright photosphere - the deepest part of the shell visible - comes tantalizingly close to the limits even of speckle interferometry. According to Paul Murdin, of the Royal Greenwich Observatory (RGO) in Sussex, spectral lines of hydrogen and helium have been detected from the shell, which is expanding at $16,000 \mathrm{~km}$ $\mathrm{s}^{-1}$, but the wide spread of relative gas velocities is for the time being smearing out most of the interesting spectral features beyond recognition. This is no doubt particularly frustrating for those studying a phenomenon that makes supernovae unique and crucial for an understanding of the chemical evolution of the Galaxy the ejection of heavy elements into the interstellar medium, including not only the residues of the star's nuclear cycle but also products such as short-lived isotopes of nickel and cobalt and stable heavy elements synthesized only during such explosions. Clearly, the neutrino detection is particularly exciting in this context.

Meanwhile, intriguingly, nobody has yet convincingly shown theoretically that supernovae should occur at all. Everybody seems happy that the inert iron core left at the end of a star's nuclear life should, if the star is sufficiently massive, undergo a phase during which electrons are captured by nuclei, thereby removing the capacity of the core to resist the weight of the overlying stellar material and producing a rapid implosion. The core soon reaches nuclear densities and the implosion stops, but nobody has been able quantitatively to explain what happens next. The hand-waving idea is that a shock-wave propagates outwards and expels the outer layers. Specifically, according to Martin Rees of the Institute of
Astronomy in Cambridge, neutrinos are released which should be the agents of such disruption, but their mean free paths are too long to be modelled as 'simple' shocks. Some calculations end with a black hole into which the entire star collapses, while others predict a neutron star remnant with outer gaseous layers whose behaviour cannot be well predicted.

What all this means in practice is that everyone is going to want to see what 1987a leaves behind. A hot neutron star would be detectable by its emitted X-rays, although for the first few months these would be obscured by emissions from the scattered energetic particles within the shell. Moreover, according to X-ray astronomers working with the Astro-C/Ginga satellite, observations will be greatly complicated by the nearby bright X-ray source LMC X-1.

The dynamics as well as the nature of the object left behind will absorb much attention - if a neutron star, will it have a high transverse velocity and will it rotate so rapidly as to act as a pulsar?

As if all this were not compelling enough, the supernova also provides an opportunity to explore the dynamics and the history of the material lying along the line of sight. Already, according to Max Pettini of the RGO, absorption lines of calcium, sodium and magnesium have revealed at least twenty distinct gas motions, many within the LMC and others within our own Galaxy. But the brightness of the object should also allow the detection of comparatively weak absorption features due to trace isotopes whose abundances constrain theories of primordial nucleosynthesis (such as ${ }^{7} \mathrm{Li}$ ) or galactic chemical evolution (such as ${ }^{13} \mathrm{C} /{ }^{12} \mathrm{C}$ ). Previous studies of this kind have been confined to the region within 300 light years or so of the Sun, but now there seems to be a prospect of similar observations within the LMC. The point is especially intriguing because the LMC is thought to be chemically much younger than our Galaxy.

So most astronomers are understandably delighted, but a small sub-group of them, doubtless now impotently furious, has already missed its only chance to exploit this event. The most aspherical type of core implosion to produce 1987a would also have generated gravitational waves. Maddeningly, no cryogenic detectors were operating but two bar detectors at the University of Maryland were taking data yet to be examined. Philip Campbell 